\title{
LOW COST 50 WATTS INVERTER FOR FLUORESCENT LAMP USING ELECTRONIC CHOKE CONTROLLED BY TIMER
}

\author{
Babu Naik G' ${ }^{1}$, T.C Balachandra ${ }^{2}$, Ozwin Dsouza ${ }^{3}$, Manjunatha Babu $\mathbf{P}^{4}$ \\ 1, 2,3,4 Department of Electrical \& Electronics Engineering, BMS Institute of Technology (VTU), Bangalore, India \\ babu.naik205@gmail.com,ozwindsouza@gmail.com
}

\begin{abstract}
Inverters are devices which can convert electrical energy form DC to AC. They are available in various sizes and in different VA ratings, from low power functions such as powering a car radio to that of backing up a building in case of power outage. In this work an attempt has been made in designing a low cost, low Power Inverter using Electronic Choke Controlled by Timer Circuit. This gives the backup during power failure and also provides a sophisticated user access. The purpose of this DC/AC inverter is typically to take $D C$ power supplied by a battery, to light a fluorescent lamp of 50 watts operating at $50 \mathrm{~Hz}$, emulating the power available at an ordinary household electrical outlet. By suitably adjusting the timer control parameters, user can turn ON/OFF of lamp automatically. The timer management is very essential for any organization or institution. Most of them usually divide the functional day into segments of working hours and other activities. In educational institutions the working hours are again divided into periods. Most of them use alarms to indicate the beginning or the end of a time segment. This is a repeating process and may rarely change day to day, so it justifies that this process could be automated.
\end{abstract}

Keywords: Fluorescent Lamp, Electronic Choke, Timer

\section{INTRODUCTION}

Inverters are used for many applications in low voltage DC sources such as batteries, solar panels, fuel cells has been be converted so that devices can run off of AC power. Our objective and approach towards this project was to design such an inverter which is of 50 Watts output working according to timer pulses and operate a fluorescent lamp in a manner of ON/OFF strategy. Here, method in which the low voltage DC power is inverted is completed in two steps [3]. The first method was the conversion of the low voltage DC power to a high voltage DC source, and the second method being the conversion of the high DC source to an AC waveform using pulse width modulation [1], [2]. This project focused on the first method described and specifically the transformation of a high voltage DC source into an AC output. The Timer unit that controls the fluorescent lamp is designed to provide the user the ease of use as well as maximum features. It is having a menu driven software along an alphanumeric LCD allows for easy machine man interface as well as a 4-Key controlled keyboard.

\subsection{The Fluorescent Lamp}

Fluorescent materials play a major role in the light sources. There are three main parts of the fluorescent lamps, like: cold cathode, hot cathode and electroluminescent. In fluorescent lamp light is emitted by exciting phosphor atoms upon via electrons. In this paper main we will be concentrating on the cold and hot cathode. Electroluminescent lamps are using "fluorescence" but they are different. The 'fluorescent lamp' we will be designed with a lamp of glass discharge tube and will be coating inside. Induction lamps are a part of fluorescent lamps but they do not have electrodes.

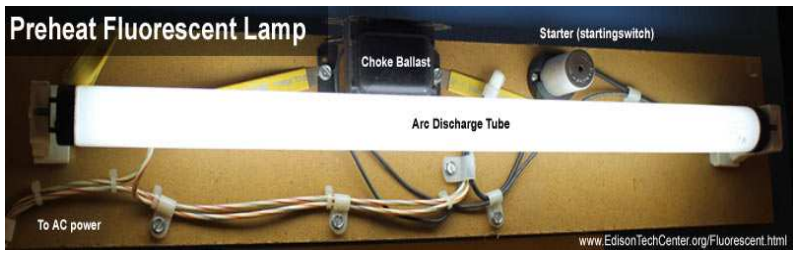

Fig.1 Typical Fluorescent Lamp

The most common fluorescent lamp is the hot cathode: it has consists of a glass tube and filled with a inert gas (argon) having low pressure. It has tungsten electrodes on each side of the tube. The "ballast" works as a regulated AC supply through the electrodes. Older lamps are used to get a starter to glow the lamp.

The standard 4 foot long 50 watt straight tube lamps of working: the AC current is flowing through the ballast and will be step up to 120 - 260 volt AC, after that power passing through the "choke" or a "reactor", this will be limiting the current and act as a prevent the lamp by creating a short circuit type which would be destroy the lamp [5]-[7]. The lamp's glass 
tube is called discharge tube and by having electrons which will pass from one end electrode to other end electrode and it is called 'arc'. To start the lamp you required a spike of high voltage to get the arc started, and the high voltage forces gives current flowing through the argon gas. For colder lamp you need high voltage to get a start, has a high resistance and therefore you required a higher voltage with colder temperatures.

This method is the first and arguably the most reliable type of way to start a lamp according to some. In the olden-days systems the starter contained a small neon or argon lamp. When the starter was cool, current ran through the starter switch through the neon lamp. The $1 \mathrm{~W}$ lamp would warm a bimetallic strip in the starter, while in the main arc tube the current passed through the tungsten electrodes which would make them heat up and ionize some of the gas. This 'preheated' the lamp. Current passes through the tungsten electrodes on each end of the lamp.

\subsection{Electronic Choke}

The Electronic ballast uses for solid state electronic circuitry to provide the proper starting lamp and operating electrical conditions to power discharge lamps. Electronic ballast either can be smaller and lighter than a comparably-rated magnetic one. The ballast has been filled with a resin to protect the circuit boards and components from moisture and vibration. Electronic ballast is usually quieter than a magnetic one, which produces a line-frequency hum by vibration of the transformer lamination. Electronic ballasts are often used based on the SMPS topology, it will rectifying the input power source and then chopping it at a high frequency. Advanced electronic ballasts may allow dimming via pulse-width modulation or via changing the frequency to a higher value [2], [3]. Ballasts have a microcontroller (digital ballasts) may offer remote control and monitoring networks such as simple analog control using a 0 to $10 \mathrm{~V}$ DC brightness control signal.

Electronic ballasts usually supply power to the lamp at a frequency of $20 \mathrm{KHz}$ or higher, rather than the mains frequency of $50 \mathrm{~Hz}$ to $60 \mathrm{~Hz}$; this eliminates the stroboscopic effect of flicker, a product of the line frequency formed with fluorescent lighting. The high output frequency of the electronic ballast refreshes the phosphors in a fluorescent lamp so that there is no perceptible flicker. The flicker index is used for measuring perceptible light modulation and ranges from 0 to 1 , with ' 0 ' meaning is lower possibility of flickering and ' 1 ' indicates the highest flickering. Lamps operated based on magnetic ballasts and has a flicker index between 0.04 to 0.07 , for digital ballasts have a flicker index of below 0.01. Because of more gas ionized in the arc stream and the lamp operates at about $9 \%$ higher efficacy above approximately $10 \mathrm{KHz}$. With the higher efficiency of the ballast and the higher lamp efficacy at higher frequency, electronic ballasts offer higher system efficacy for low pressure lamps like the fluorescent lamp.

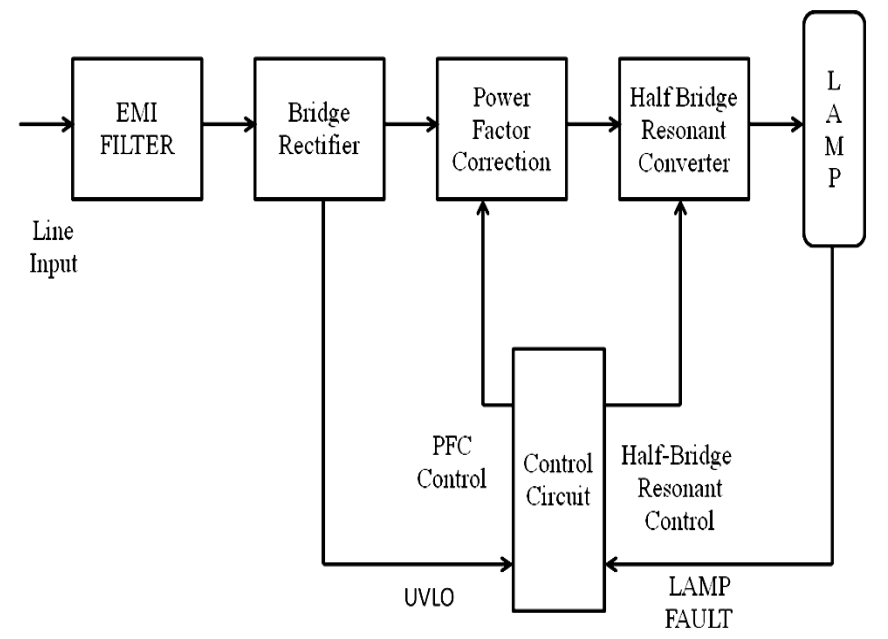

Fig.2 Block Diagram of fluorescent lamp mechanism

\section{TIMER}

Time management is very essential for any organization or institution. Most of them usually divide the functional day into segments of working hours and other activities. In educational institutions the working hours are again divided into periods. Most of them use alarms to indicate the beginning or the end of a time segment. A person in charge switches or rings the bell. This is a repeating process and may rarely change day to day, so it justifies that this process could be automated. The proposed timer is basically meant to do the above mentioned. The unit is designed to provide the user the ease of use as well as maximum features. A menu driven software along an alphanumeric LCD allows for easy machine man interface. The unit can be programmed for a maximum of twenty bell settings, each setting being in HH:MM format, and the time entered is in twenty-four hour mode. An onboard RTC maintains the clock calendar. The RTC is backed by a lithiumion battery, which ensures valid time and settings even at power down [6]. It is interfaced to an Atmel controller, which is the heart of the circuit. The controller is a 20 pin device which is a scaled down device having an 8051 core with builtin $2 \mathrm{~K}$ Bytes of flash memory [4]. A simple 4-Key keyboard allows the user to operate the unit. The bell itself is switched by a relay. The user could set bell settings, change current time, delete settings, and change access code. The bell settings are continuously compared with the RTC time and appropriate action taken. The bell can be manually switched ON or OFF using one of the keyboard keys. When not in set mode the unit displays the current time/date. 


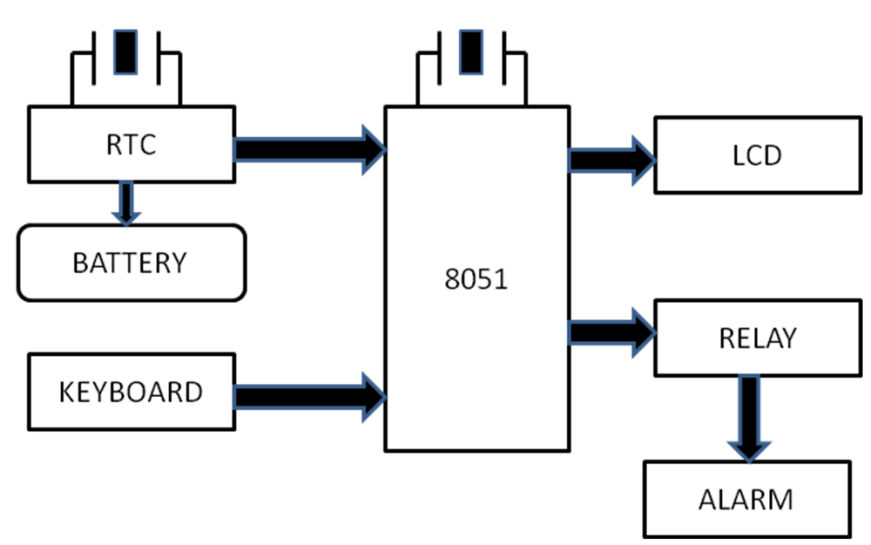

Fig.3 Block Diagram of Timer

\subsection{Description}

The AT89C2051 is a low-voltage, high-performance CMOS 8bit microcomputer with $2 \mathrm{~K}$ Bytes of Flash programmable and erasable read only memory (PEROM). By combining a versatile 8-bit CPU with Flash on a monolithic chip, the Atmel AT89C2051 is a powerful microcomputer which provides a highly flexible and cost effective solution to many embedded control applications. The AT89C2051 provides the following standard features: 2K Bytes of Flash, 128 bytes of RAM, 15 I/O lines, two 16-bit timer/counters, a five vector two-level interrupt architecture, a full duplex serial port, a precision analog comparator, on-chip oscillator and clock circuitry. In addition, the AT89C2051 is designed with static logic for operation down to zero frequency and supports two software selectable power saving modes. The Idle Mode stops the CPU while allowing the RAM, timer/counters, serial port and interrupt system to continue functioning. The Power down Mode saves the RAM contents but freezes the oscillator disabling all other chip functions until the next hardware reset. Basic Features of the Chip:

- Compatible with MCS-51 Products

- $2 \mathrm{~K}$ Bytes of Flash Memory

- $\quad 2.7 \mathrm{~V}$ to $6 \mathrm{~V}$ Operating Range

- Fully Static Operation: $0 \mathrm{~Hz}$ to $24 \mathrm{MHz}$

- Two-Level Program Memory Lock

- 128 x 8-Bit Internal RAM

- 15 Programmable I/O Lines

- Two 16-Bit Timer/Counters

- Six Interrupt Sources

- Programmable Serial UART Channel

- Direct LED Drive Outputs

- On-Chip Analog Comparator

- Low Power Idle and Power Down Modes

\subsection{Internal Architecture of Timer}

\begin{tabular}{|l|l|}
\hline Port Pin & Alternate Functions \\
\hline P3.0 & RXD (Serial Input Port) \\
\hline
\end{tabular}

\begin{tabular}{|l|l|} 
P3.1 & TXD(Serial Output Port) \\
\hline P3.2 & INT0 (External Interrupt 0) \\
\hline P3.3 & INT1 (External Interrupt 1) \\
\hline P3.4 & T0 (Timer 0 External Input) \\
\hline P3.5 & T1 (Timer 1 External Input) \\
\hline
\end{tabular}

Table 1: Port Pin Function

Port 1 is an 8-bit bidirectional Input/output port. Port pins P1.2 to $\mathrm{P} 1.7$ provide internal pull-ups. $\mathrm{P} 1.0$ and $\mathrm{P} 1.1$ are requiring external pull-ups. P1.0 and P1.1 also has the positive input and the negative input, of the on-chip precision analog comparator. The Port 1 output buffers of sink $20 \mathrm{~mA}$ and will be drive LED displays directly. When $1 \mathrm{~s}$ is written to Port 1 pins and can be used as inputs. When pins P1.2 to P1.7 are used for inputs and work as externally pulled low, they have source current because to provide the internal pull-ups. Port 1 is receives code data during Flash programming and verification. Port 3 pins P3.0 to P3.5, P3.7 has seven bidirectional Input/output pins are with internal pull-ups. P3.6 has hard-wired from input to the output of the on-chip comparator and is not accessible as a general purpose Input/output pin. The Port 3 output buffers a sink of $20 \mathrm{~mA}$. When $1 \mathrm{~s}$ is written to Port 3 pins have the pulled high by the internal pull-ups and can be used as inputs. From inputs of the Port 3 pins that are externally used as a pulled low will source current because of it has the pull-ups.

- Reset input (RST): All Input/output pins are reset to 1s when the RST goes to high level. Holding the RST

- $\quad$ pin is high for two machine cycles while the oscillator is running resets the device. Each machine cycle takes as a 12 oscillator/clock cycles.

- XTAL1: from the Input the inverting oscillator amplifier and the internal clock operating circuit to the input.XTAL2: the inverting oscillator amplifier the output.

\subsection{Serial Real-Time Clock:}

The DS1307 Serial Real-Time Clock is a low-power, full binary-coded decimal (BCD) of the clock/calendar plus 56 bytes of NV SRAM. Address and data are transferred with a sequence via a 2-wire to bidirectional bus. The clock/calendar has to provide the seconds-minutes-hours-day-date-month-year information. The end of the month date is automatically adjusted for months of the 31 days, including the corrections for leap year. The clock operates in either the 24-hour/12-hour format with AM/PM indicator. The DS1307 has been built in power sense circuit of models to detect the power failures and automatically switches-ON to the battery supply.

The DS1307 operates as a slave device of the serial bus. Access is gained by making a START condition and providing a device identification code followed by a register address. 
Subsequently registers can be accessed periodically until a STOP condition is executed. When VCC falls below 1.25VBAT the device terminates an access in progress and resets the device of address counter. From Inputs the device will not be recognized at this condition to prevent erroneous data from being written to the device for the out of tolerance system. When VCC falls below VBAT and the device switches into a low-current battery to make a backup mode. When the powerup, the device switches from battery to VCC when VCC is greater than VBAT $+0.2 \mathrm{~V}$ and recognizes inputs when $\mathrm{VCC}$ is greater than 1.25 of the VBAT.

\subsection{RTC and RAM address MAP}

The address map for the RTC and RAM registers of the DS1307 is shown in Figure. The RTC registers are located in address locations $00 \mathrm{~h}$ to $07 \mathrm{~h}$. The RAM registers are located in address locations $08 \mathrm{~h}$ to $3 \mathrm{Fh}$. During a multi-byte access, when the address pointer reaches $3 \mathrm{Fh}$, the end of RAM space, it wraps around to location $00 \mathrm{~h}$, the beginning of the clock space.

\begin{tabular}{|c|}
\hline (00H) SECONDS \\
\hline MINUTES \\
\hline HOURS \\
\hline DAY \\
\hline DATE \\
\hline MONTH \\
\hline (07H) YEAR \\
\hline$(08 \mathrm{H}) \mathrm{CONROL}$ \\
\hline $\begin{array}{l}\text { RAM } \\
(3 \mathrm{FH})\end{array}$ \\
\hline
\end{tabular}

Table: 2 DS1307 Address Map

\subsection{Clock and Calendar}

The time and calendar information is obtained by the appropriate register bytes. The time and calendar are set/ initialized by the appropriate register bytes. The indexes of the time and calendar registers are in the $\mathrm{BCD}$ format. Bit 7 of register 0 is the clock halt $(\mathrm{CH})$ bit. If the bit is set to ' 1 ', the oscillator is disabled and it's cleared to ' 0 ' the oscillator is enabled. The DS-1307 can be work in either 12-hour/24-hour mode. Bit 6 of the hours register is defined as the 12-hour/24hour mode select bit. If it is high, the 12 -hour mode will be selected. In the 12-hour mode bit 5 will be selected and the AM/PM bit with logic code has high PM. In the 24-hour mode, bit 5 is the second 10 hour bit. On a 2-wire START, the current time is transferred to a second set of registers. The time information is read from these secondary registers, while the clock may continue to run. This eliminates the need to reread the registers in case of an update of the main registers during a read.

\subsection{Control Register}

\begin{tabular}{|l|l|l|l|l|l|l|l|}
\hline BIT7 & BIT6 & BIT5 & BIT4 & $\begin{array}{l}\text { BIT } \\
3\end{array}$ & BIT2 & BIT1 & BIT0 \\
\hline OUT & 0 & 0 & $\begin{array}{l}\text { SQW } \\
\text { E }\end{array}$ & 0 & 0 & RS1 & RS0 \\
\hline
\end{tabular}

Table 3: Control Register

Output control (OUT): This bit has the controlling the output level of the SQW or OUT pin where as square wave output is disabled. If SQWE $=0$, the logic level of the SQW/OUT pin is 1 if $\mathrm{OUT}=1$ and the logic level is 0 if $\mathrm{OUT}=0$.

Square Wave Enable (SQWE): This bit, when set to logic 1, the oscillator of the output will enable. The frequency of the square wave output depends upon the value of the RS-0 and RS-1 bits. With the square wave output will be set to the $1 \mathrm{~Hz}$, the clock registers will be updated and falling edge of the square wave.

RS (Rate Select): These bits control the frequency of the square wave output when the square wave output has been enabled. Table lists the square wave frequencies that can be selected with the RS bits.

\begin{tabular}{|l|l|l|}
\hline RS1 & RS0 & SQW OUTPUT FREQUENCY \\
\hline 0 & 0 & $1 \mathrm{~Hz}$ \\
\hline 0 & 1 & $4.096 \mathrm{KHz}$ \\
\hline 1 & 0 & $8.192 \mathrm{KHz}$ \\
\hline 1 & 1 & $32.768 \mathrm{KHz}$ \\
\hline
\end{tabular}

Table 4: Square wave output frequency

\subsection{Display and Keyboard Interface:}

The LCD used is a alphanumeric 16 characters X 1 line. The display has a back light, which helps when the unit is used in dark. The LCD is in fact divided as $8 \times 2$ lines, so the software has to suitably interpret. The LCD is an asynchronous device. The display is updated by writing ASCII values. The 4-bit bus is connected to Port 0 of the processor. There are physically two registers inside the display unit, those are Command register and Data register. These can be selected by applying the appropriate logic on the RS (register select) pin. A high selects Command register and a low the Data register. A read or write to these registers is accomplished through the R/W pin. The Enable pin controls the selection of display, which is active high. The Command register should be read to check whether the display is busy. If the LCD is busy with its internal 
operations the next character or command should not be sent. However the maximum time period consumed for the longest operation is 40us, so the software before every action pauses of a bit more than 40us. As the Busy status is not read the R/W pin is permanently connected to ground which puts the display always in write mode. Pin 1 is ground, Pin 2 is power and is connected to Vcc. Pin 3 is brightness control which is connected to a variable resistor $\mathrm{R} 2$, so the user can set the brightness to his taste. The LCD has to be initialized first before writing data into it. The keyboard circuit is very straight forward. The upper four lines of port $\mathrm{p} 0$ is connected to one end of four keys whose other end is connected to ground, The Keyboard lines are pulled high through R7, so when none of them are pressed the processor reads a logical high on all the lines. On pressing of any key the corresponding line goes low indicating the processor of the press.

\section{SCOPE OF THE PRESENT WORK}

\subsection{Statement of Project}

This paper focuses on DC to AC 50 Watts inverter, which aim to efficiently transform a DC power source to an AC source and a timer circuit with an LCD display and a 4-Key controlled keyboard controlling the operation of a Fluorescent Lamp.

\subsection{Specific Objectives}

- To create an inverter of 50 Watts output to light a fluorescent lamp.

- To design a timer with LCD display to control the fluorescent lamp.

- To implement an additional feature to inverters that is, controlling any device in A.C. power off mode with respect to time.

- To learn Microcontroller Programming using Keil $\mu$ Vision 4 which was very interesting and resulted in productiveness.

\subsection{Present Work}

In the present situation the available efficient highs rating Inverters is not cost effective and are complex in implementation. Keeping this in mind our aim is to build an efficient and a low rating inverter which will light a fluorescent lamp and operate according to timer connected with it. Also, it is built with simple circuit components.

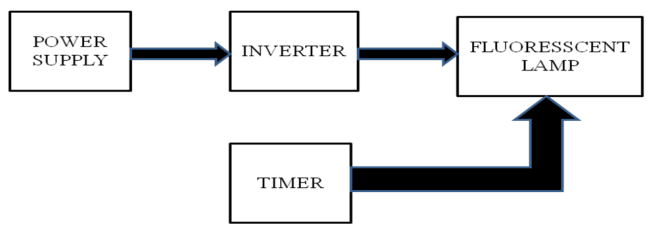

Fig.4: Block Diagram of Inverter lighting a fluorescent Lamp using a Timer control

\subsection{Working of an Inverter}

Here is a 50 Watt inverter circuit using minimum number of components. The circuit diagram of a simple 50 watt inverter is using IC CD4047 and MOSFET IRF44. It is quite difficult to make a decent one like this with further fewer components. Here we are using CD 4047 IC from Texas Instruments for generating the $50 \mathrm{~Hz}$ pulses and four $2 \mathrm{~N} 3055$ transistors for driving the load. The IC1 Cd4047 wired as a stable multivibrator produces two 180 degree out of phase $50 \mathrm{~Hz}$ pulse trains. These pulse trains are preamplifier by the two TIP122 transistors. The out puts of the TIP 122 transistors are amplified by four $2 \mathrm{~N} 3055$ transistors (two transistors for each half cycle) to drive the inverter transformer. The $220 \mathrm{~V} \mathrm{AC}$ will be available at the secondary of the transformer. Nothing complex just the elementary inverter principle and the circuit works great for small loads like a few bulbs or fans.

\subsection{The Timer Operations:}

The design based on a microcontroller enhances the final outlook of the instrument in terms of its operation and ease of programming:

A discreet digital circuit even if designed to do the same functionality will never be user friendly in terms of user interaction

The microcontroller along with a simple keyboard and a 16X1 alphanumeric display makes life easier for the user. When the unit is powered up it displays "Timer" for 2 seconds and then displays the current time.

The display is updated every second. At this moment the user can toggle the display between time and date by pressing the <Ent> key. The complete date in the format dd:mm:yyyy is displayed. The bell is usually switched on automatically by the unit when the current time equals the setting time.

It can be manually switched on by pressing the $\langle$ Inc $\rangle$ key and can be switched off by pressing the same key. The above mentioned keys are the two direct function keys the rest of the functionality is to be done by pressing the $\langle$ Fnc $\rangle$ key. Upon pressing the $\langle$ Fnc $\rangle$ key the user is presented with a menu item, if he wishes to select it he has to press <Ent> or <Fnc $>$ to proceed to next menu item.

Once when a item is selected and changes made the menu can be quit by saving changes by pressing <Ent> or quitting the menu item without saving changes by pressing $\langle$ Fnc $\rangle$.Pressing $\langle$ Fnc $\rangle$ for the first time the user is asked for an access code which has to be entered using $\langle\mathrm{Inc}\rangle$ and $\langle\mathrm{Dec}\rangle$ keys. Pressing $<$ Ent $>$ the entered code is validated and if correct the first menu item "Bell Settings". 
Pressing <Ent> the display changes to "No.of Settings". This is the number of times the bell has to be ring. The value on the display can be changed using inc and dec keys.

\subsection{Hardware Design}

1. The Full Project Design

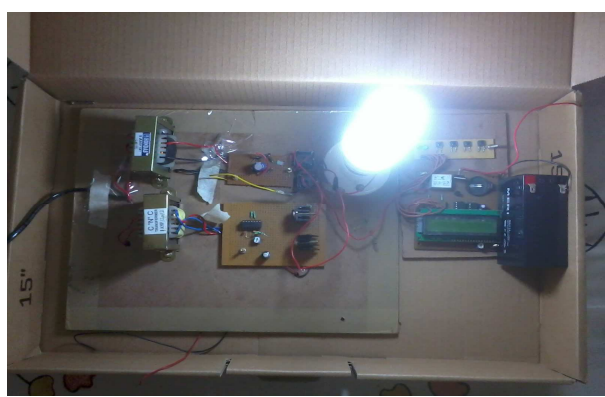

Fig.5: The Complete Project Design

2. The Timer connected to a 12 V D.C. Source.

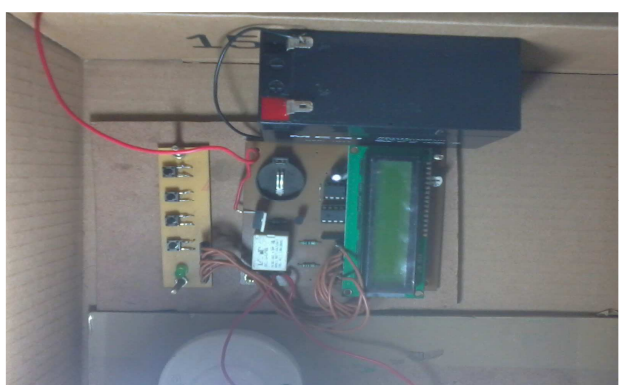

Fig.6: The Timer connected to a 12V D.C. Source

3. The timer Bell setting and time setting.

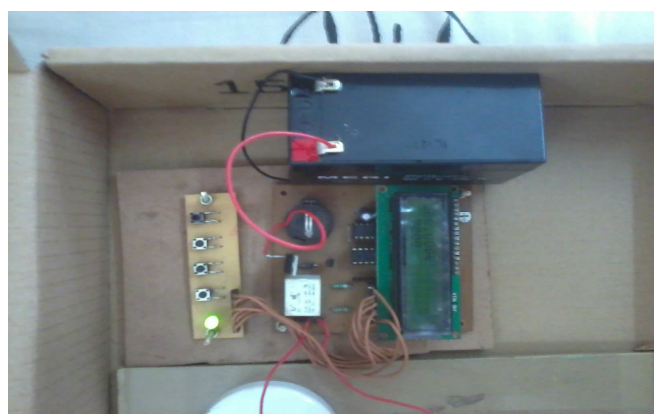

Fig.7: The timer Bell setting and time setting.

\section{RESULTS AND DISCUSSIONS}

Our project deals with a 50 watt inverter which will light a fluorescent lamp controlled by a timer. Inverter is the heart of this project we have made a 50 watt inverter using IC Cd4047,
MOSFET IR44, 2N3055 transistor and TIP122 transistor. IC $\mathrm{Cd} 4047$ is an a stable multivibrator, the $50 \mathrm{~Hz}$ pulse trains of IC Cd4047 are preamplified by the two TIP122 transistors. The output of TIP122 transistors are amplified by four 2 N3055 transistors. A Timer is used in this project which is controlled by a Microcontroller AT89C2051 and a real time clock IC DS1307 and it has LCD display as well as 4-Key controlled keyboard. A fluorescent lamp is switched on and off according to the timer control and the inverter lights up the fluorescent lamp.

\section{CONCLUSIONS}

The goals for this project were to produce a pure sine wave DC/AC inverter that would output at $50 \mathrm{HZ}, 230 \mathrm{~V} \mathrm{AC}$, to light a 50 watt fluorescent lamp would be cheap to manufacture, and fairly efficient in the method in which it produces it. The second goal was to light a fluorescent lamp of 50 watts according to timer controlled operation.

\section{REFERENCES}

[1] Wenyi Zhang, Wensheng Chen, "Research on voltagesource PWM inverter based on state analysis method" in Mechatronics and Automation, International Conference on Digital Object Identifier, 2009. ICMA 2009, Page(s): 2183 2187

[2] Khluabwannarat.P, Thammarat.C, Tadsuan. S, Bunjongjit. $\mathrm{S}$, "An analysis of iron loss supplied by sinusoidal, square wave, bipolar PWM inverter and unipolar PWM inverter" in Power Engineering Conference, 2007. IPEC 2007. International Publication Year: 2007 , Page(s): 1185 - 1190.

[3] Femia. N, Vitelli.M, "Frequency domain analysis of DCDC converters with internally controlled switching instants" Power Electronics Congress, 1996. Technical Proceedings. CIEP '96., V IEEE International Digital Object Identifier: 10.1109/CIEP.1996.618551, 1996 , Page(s): 269 - 276

[4] Deitrich.B, "Microprocessor modeling in VHDL without a gate/RTL/behavioral model of the microprocessor" Military Communications Conference, 1992. MILCOM '92, Conference Record. Communications - Fusing Command, Control and Intelligence. IEEE, Digital Object Identifier: 10.1109/MILCOM.1992.243962, 1992 , Page(s): 975 - 978 vol.3

[5] Mohan, Undeland, Riobbins, "Power Electronics Converters Applications and Design" Wiley India Edition, Third Edition 2009.

[6] M. H. Rashid, "Power Electronics - Circuits, Devices and Applications," Prentice-Hall Inc., 2nd edition, 1993, 695 pages, ISBN 0-13-678996-X. 10. M. H.

[7] Albert Malvino and David J. Bates "Electronic Principles knowledge of electronics I suggest Electronic Devices and Circuit Theory" 7th Edition 


\section{BIOGRAPHIES:}

Babu Naik Gugulothu, has received his masters from the Indian Institute of Science (IISc) Bangalore, India. Currently working as Assistant professor in the department of Electrical and Electronics Engg at BMSIT Bangalore. His research interests are in the field of Industrial drives and applications, power systems, SMPS circuits, converter topologies and electrical machines

T.C. Balachandra received the Ph.D. degree from the Indian Institute of Science, Bangalore, India in 1995. He is currently Professor \& Head, Department of Electrical and Electronics Engineering at BMSIT Bangalore, India. His research interests include vacuum insulation, micro and smart systems technology, insulation coordination \& condition monitoring of electrical \& electronic systems.

Ozwin Dominic Dsouza has received his masters from National Institute of Engineering, Mysore, India. Currently working as Assistant professor in the department of Electrical and Electronics Engg at BMSIT Bangalore. His research interests are in the field of electric drives and systems and control systems, converter topologies and electrical machines.

Manjunathababu $\mathbf{P}$, has received his masters from UVCE, , India. Currently working as Assistant professor in the department of Electrical and Electronics Engg at BMSIT Bangalore. His research interests are in the field of electric drives and systems and control systems, converter topologies and electrical machines 